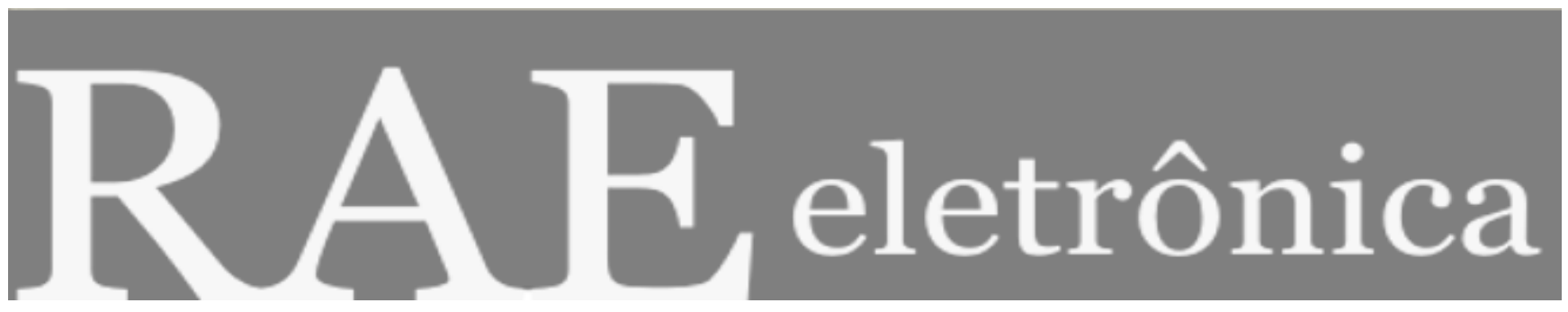

IMPLEMENTAÇÃO DE ECO-TECNOLOGIAS RUMO À ECOLOGIA INDUSTRIAL

Por:

Biagio Fernando Giannetti

Cecília M. Villas Boas de Almeida

Sílvia H. Bonilla

RAE-eletrônica, Volume 2, Número 1, jan-jun/2003.

http://www.rae.com.br/eletronica/index.cfm?FuseAction=Artigo $\& I D=1236 \&$ Secao=CIÊNCIA $\&$ Volume $=2 \&$ Numero $=1 \& A$ no $=2003$

CCopyright, 2002, RAE-eletrônica. Todos os direitos, inclusive de tradução, são reservados. É permitido citar parte de artigos sem autorização prévia desde que seja identificada a fonte. A reprodução total de artigos é proibida. Os artigos só devem ser usados para uso pessoal e não-comercial. Em caso de dúvidas, consulte a redação: redacao@,rae.com.br.

A RAE-eletrônica é a revista on-line da FGV-EAESP, totalmente aberta e criada com o objetivo de agilizar a veiculação de trabalhos inéditos. Lançada em janeiro de 2002, com perfil acadêmico, é dedicada a professores, pesquisadores e estudantes. Para mais informações consulte o site www.rae.com.br/eletronica.

RAE-eletrônica

ISSN 1676-5648

(C)2002 Editora: Fundação Getulio Vargas - Escola de Administração

de Empresas de São Paulo.

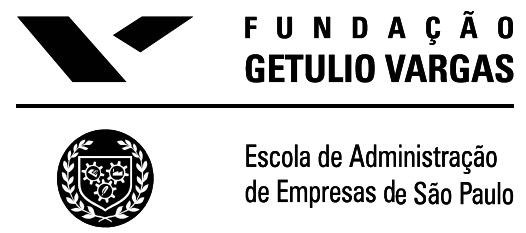




\section{IMPLEMENTAÇÃO DE ECO-TECNOLOGIAS RUMO À ECOLOGIA INDUSTRIAL}

\section{Biagio Fernando Giannetti}

Coordenador do LaFTA - Laboratório de Físico-Química Teórica e Aplicada

Coordenador do Curso de Engenharia Química da Universidade Paulista - UNIP

Professor do Programa de Pós-Graduação em Engenharia de Produção - UNIP

E-mail: biafgian@unip.br

Endereço: R. Dr. Bacelar, 1212, CEP 04026-002 São Paulo, SP

Interesses de pesquisa: Ecologia Industrial, Produção e Meio Ambiente, Físico Química, Eletroquímica

\section{Cecília M. Villas Boas de Almeida}

Pesquisadora do LaFTA - Laboratório de Físico-Química Teórica e Aplicada

Coordenadora do Curso de Engenharia Química da Universidade Paulista - UNIP

Professora do Programa de Pós-Graduação em Engenharia de Produção - UNIP

E-mail: cmvbag@terra.com.br

Endereço: R. Dr. Bacelar, 1212 - São Paulo, SP 04026-002

Interesses de pesquisa: Ecologia Industrial, Produção e Meio Ambiente, Físico Química, Eletroquímica

\section{Sílvia H. Bonilla}

Pesquisadora do LaFTA - Laboratório de Físico-Química Teórica e Aplicada

Professora do Curso de Engenharia Química da Universidade Paulista - UNIP

E-mail: biafgian@unip.br

Endereço: R. Dr. Bacelar, 1212, CEP 04026-002 São Paulo, SP

Interesses de pesquisa: Físico Química, Eletroquímica

\section{RESUMO}

Este trabalho faz uma reflexão sobre mudanças conceituais devidas ao desenvolvimento/implementação de eco-tecnologias sob a ótica da Ecologia Industrial. Inicialmente, discutem-se conceitos fundamentais da Tecnologia Mais Limpa e da eco-tecnologia. Soma-se à discussão uma revisão das tecnologias não convencionais de tratamento/prevenção à poluição existentes na literatura científica recente, de forma a traçar estratégias que permitam aplicá-las no sistema industrial na forma de Eco-Tecnologias. Utilizando exemplos de tratamento de efluentes contendo metais pesados, como efluentes de curtume, o texto discute a necessidade da inclusão da pesquisa científica no conceito de Ecologia Industrial. A contribuição do artigo é contextualizar claramente o conceito de Eco-Tecnologia empregando uma proposta de aplicação para setores industriais do sul do país. O trabalho aponta a grande responsabilidade de profissionais que podem estimular ações sinérgicas das indústrias no tratamento de resíduos como alternativa às, generalizadas, práticas isoladas das empresas.

\section{Abstract}

This work displays a reflection on conceptual changes of the development/implementation of EcoTechnology from an Industrial Ecology perspective. Initially, the fundamentals of the concepts Cleaner Technology and Eco-Technology are discussed. An extensive bibliographic review of non-conventional technologies is presented for positioning Production in a strategic context. Drawing on examples from 
heavy metal attenuation, such as chromium from tannery industry, this work discusses the need of including scientific research on the concept of Industrial Ecology, by means of the development of EcoTechnologies. This paper brings to discussion a clear definition of Eco-Technology employing a proposition for application of these technologies to industrial sectors in South Brazil. The work attributes great responsability to professionals who may estimulate sinergistic actions in industries, regarding the disposal and effluent treatment, as alternative to the present isolated practices.

\section{PALAVRAS-CHAVES}

Ecologia industrial, regional, produção mais limpa.

\section{KEY WORDS}

Industrial ecology, regional, cleaner production. 


\section{INTRODUÇÃO}

O vigoroso desenvolvimento do mercado global e da tecnologia cria grandes desafios para o setor industrial. A Produção deverá encontrar um novo rumo considerando o desenvolvimento de produto, fornecedores, distribuidores e, principalmente, o meio ambiente. Um fator importante a ser considerado é a utilização mais eficiente das matérias primas não renováveis e renováveis pelas indústrias, manufaturas e, também, pelos consumidores. Uma possível abordagem para enfrentar tal desafio é a observação dos princípios da Ecologia Industrial ${ }^{1}$, que implicam em uma visão sistêmica do meio ambiente e da atividade industrial. Da mesma forma que se empregam Tecnologias Mais Limpas ${ }^{2}$ como técnicas para implementação da Produção Mais Limpa ${ }^{3}$, neste artigo definimos Eco-Tecnologias como ferramentas para aplicação da Ecologia Industrial.

O objetivo deste trabalho é responder algumas questões fundamentais: (i) qual a relação entre as Tecnologias Mais Limpas e o novo conceito de Eco-Tecnologias? e (ii) como as tecnologias desenvolvidas em ambiente de laboratório podem se tornar Eco-Tecnologias?

Para atingir tal objetivo, este trabalho está estruturado da seguinte forma:

Na seção Alguns conceitos fundamentais, a orientação seguida foi a de apresentar, de forma simplificada, conceitos que serão utilizados no decorrer da discussão. Definições de alguns termos consagrados na literatura podem ser consultadas nas Notas no final do trabalho complementando o texto.

Na seção seguinte Cenário Atual: exemplos de Produção Mais Limpa e Tecnologias Mais Limpas ilustra-se a aplicação da filosofia proativa de Produção Mais Limpa por meio da implementação de técnicas genéricas denominadas Tecnologias Mais Limpas. Com esta finalidade, se apresentam dados da remoção de metais pesados de efluentes de curtumes.

Na seção Estudos de laboratório: possibilidades rumo às Eco-Tecnologias é apresentada um revisão bibliográfica de tecnologias não convencionais, que foram concebidas para utilização end of pipe 4 para remoção de metais de efluentes líquidos. Utilizou-se o formato de tabelas para agrupar abordagens semelhantes. Os trabalhos citados poderão servir de ponto de partida para futuras pesquisas em gerenciamento de Eco-Tecnologias. No texto, como é de praxe, são discutidos os trabalhos mais relevantes.

Na seção Proposta de aplicação, com base nos conceitos discutidos nas seções anteriores e na revisão bibliográfica apresentada, discute-se o potencial uso de resíduos (da indústria de mineração) para tratamento de resíduos da indústria de curtume. Discutindo um caso da região sul do Brasil, aponta-se a possibilidade de uma tecnologia, desenvolvida em laboratório numa abordagem end-ofpipe, ser definida como Eco-Tecnologia quando inserida no conceito de Ecologia Industrial. Finaliza-se a seção discutindo os benefícios e as dificuldades da implementação de Eco-Tecnologias.

Finalmente, na conclusão, são respondidas às questões propostas na introdução do trabalho.

\section{Alguns conceitos fundamentais}

Cabe ressaltar que estes conceitos são relativamente novos e que, em alguns casos, as definições se interceptam e/ou se sobrepõem. Desta forma, algumas opções, como limitações ou valorizações de algumas características, podem ser consideradas preferência dos autores. 
A aplicação de conceitos de Produção Mais Limpa implica na diminuição de resíduos/rejeitos gerados e no desenvolvimento de um produto que cause menor impacto ambiental no final de seu ciclo de vida. Neste caso, o fabricante controla todos os estágios da vida do produto, incluindo a prémanufatura que pode ser influenciada pela interação fabricante/fornecedor. Sob esta abordagem, quatro etapas podem ser propostas. Em primeiro lugar substituir matérias primas considerando o significado ambiental da utilização de matérias primas não renováveis. Em seguida, observar a necessidade de melhorar o processo de manufatura, definir a real necessidade de insumos e estabelecer a viabilidade da reutilização/reciclabilidade de sub-produtos. Em uma terceira etapa, as implicações ambientais de embalagem e distribuição do produto devem também ser consideradas e, finalmente, o produto em si não deve ser classificado como produto final, mas sim como um intermediário que pode ser reutilizado ou reciclado no final de sua vida útil.

O conceito pioneiro de Produção Mais Limpa contribuiu para a otimização de processos industriais sob o ponto de vista ambiental. A Figura 1A mostra uma unidade industrial convencional que considera não haver limitação para entrada de matéria prima ou energia. A geração de resíduo é alta e, mesmo após o tratamento, é descartada totalmente no meio ambiente. A aplicação dos conceitos de Produção Mais Limpa resulta em melhor aproveitamento de matéria prima e energia (Figura 1B). A implementação de processos de reutilização/reciclagem e produção de sub-produtos vendáveis aumenta o fluxo de materiais dentro da unidade industrial e tem como consequência a diminuição da geração de resíduo.

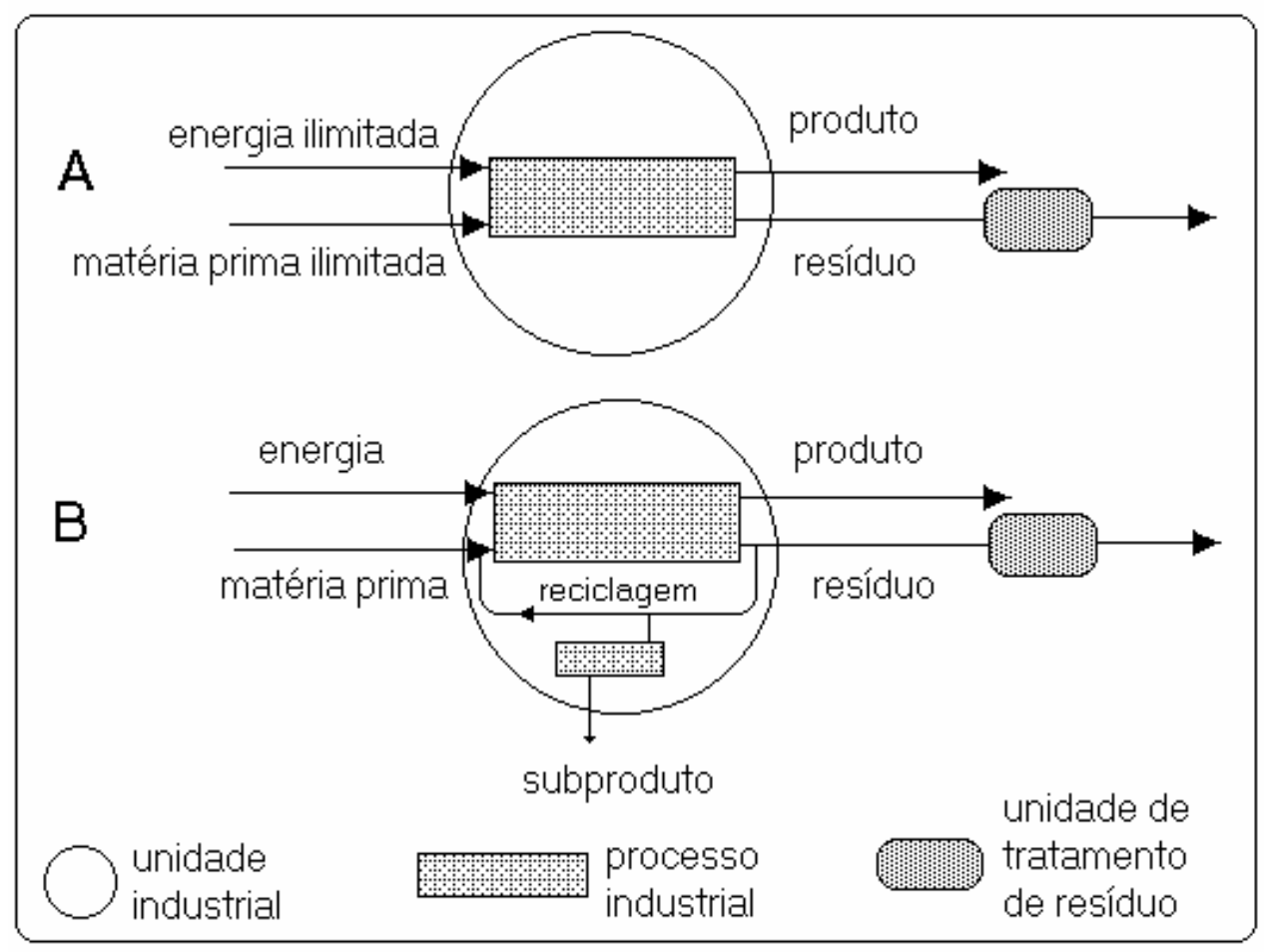

Fig 1. Representação esquemática de uma empresa convencional (A) e um empresa onde são aplicados conceitos de Produção Mais Limpa (B). 


\section{CIÊNCIA E TECNOLOGIA - IMPLEMENTAÇÃO DE ECO-TECNOLOGIAS RUMO A ECOLOGIA INDUSTRIAL}

Biagio Fernando Gianetti - Cecília M. Villas Boas de Almeida - Silvia H. Bonilla

Por outro lado, se a utilização de Tecnologias Mais Limpas, apesar de gerar benefícios econômicos e ambientais, ficar restrita a uma única empresa, permanecerá limitada: a Produção Mais Limpa, da forma como foi concebida, falha ao tentar atingir o Desenvolvimento Sustentável ${ }^{5}$ (Wallner, 1999). Entretanto, estes conceitos continuam evoluindo e se aprimorando.

A Figura 2 mostra um esquema onde se representa um conjunto de unidades industriais que se interligam para formar unidades maiores, como parques industriais e clusters. A complexidade do conjunto pode aumentar em vários níveis, por exemplo: no número e na diversidade de unidades industriais e na quantidade de interações dentro do conjunto. Uma empresa pode comprar resíduo de mais de um fornecedor, ou pode vender seus resíduos e/ou sub-produtos para mais de uma companhia.

O conceito de Ecologia Industrial, embora não explicitamente, é encontrado na literatura desde os anos setenta. A grande aceitação do termo no meio empresarial se deve à forte analogia estabelecida, entre sistemas naturais e sistemas produtivos, por dois executivos da General Motors, no artigo "Strategies for Manufacturing" (Frosh e Gallopoulos, 1989). Este conceito vem ganhando a atenção dos grupos de pesquisa em Produção Mais Limpa e o debate sobre os rumos do desenvolvimento industrial tem se renovado sob esta nova ótica. A observação dos conceitos de Ecologia Industrial leva ao exame integrado das interações entre a indústria e o meio ambiente. O sistema industrial é considerado como produtor tanto de produtos como de resíduos. Com esta abordagem, os limites de uma empresa se estendem até o meio ambiente, exigindo que produtos e resíduos sejam desenvolvidos e discutidos entre diferentes empresas. O aspecto mais crítico deste novo conceito é, portanto, a implementação da cooperação efetiva entre empresas (veja possíveis interação na Figura 2).

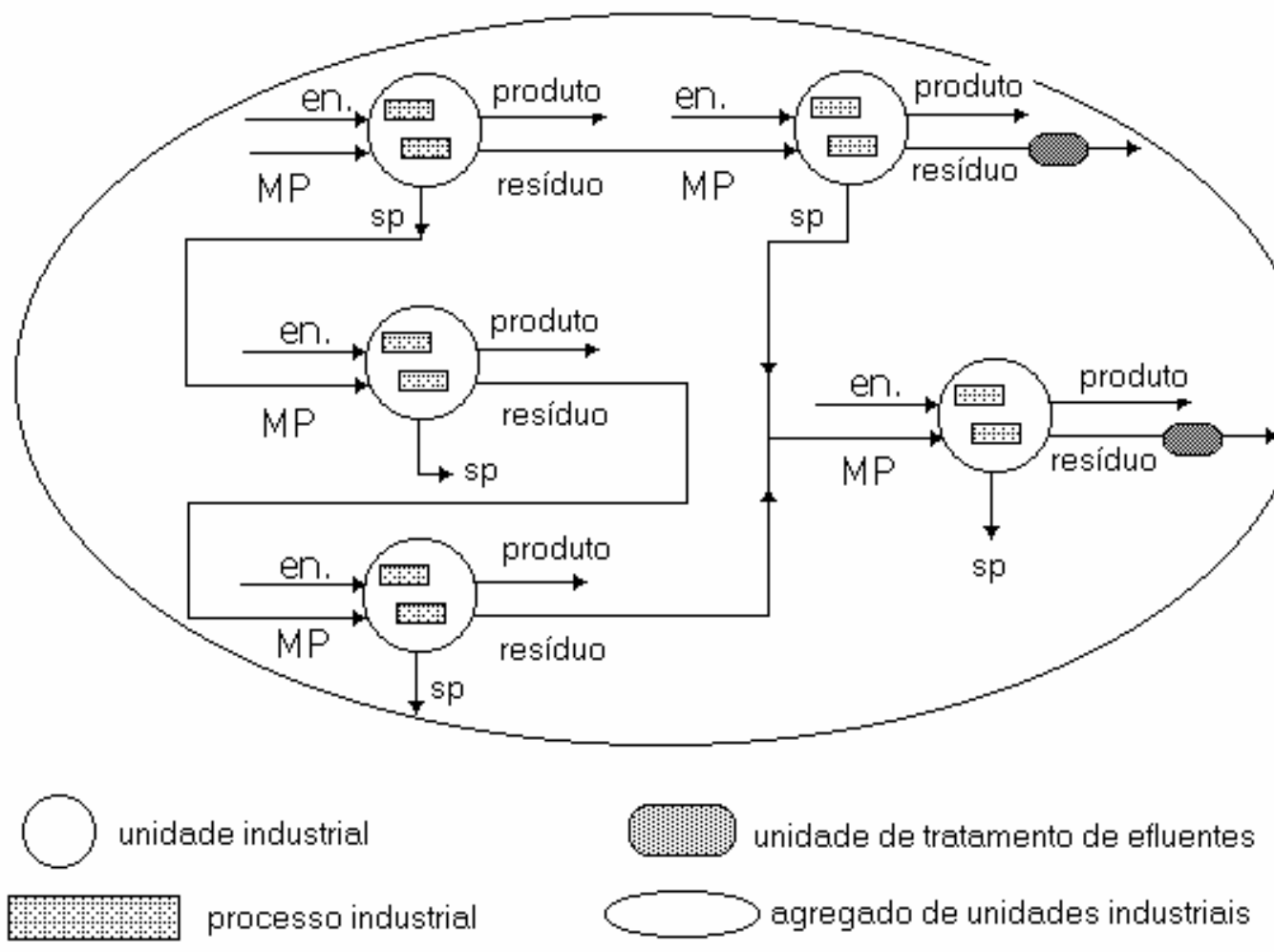

Fig. 2. Representação de um agregado de unidades industriais mostrando possíveis interações entre unidades, onde nos fluxos (en) é energia, (MP) matéria prima e (sp) subproduto. 
É importante mencionar que, durante os anos oitenta, surgiu também a metáfora "Metabolismo Industrial", especialmente devida ao trabalho de Ayres et al. (1989). Entretanto, uma distinção deve ser feita. O Metabolismo Industrial trata dos fluxos de matéria e energia no sistema industrial. Esta abordagem visa o entendimento da circulação de materiais e dos fluxos de energia desde a extração da matéria prima até o final do ciclo de vida do produto. A Ecologia Industrial vai mais longe. A abordagem procura entender como o sistema industrial opera, quais são os mecanismos reguladores e qual sua interação com a biosfera. Desta forma, com base no conhecimento dos ecossistemas naturais, pretende determinar como o sistema industrial pode ser reestruturado para torná-lo compatível com o meio ambiente.

O objetivo ideal da Ecologia Industrial é produzir resíduo ZERO, ou seja usar/re-usar totalmente todas as matérias primas disponíveis, como acontece nos ecossistemas naturais. Este conceito surgiu como resposta dos profissionais da indústria ao grande impacto negativo causado ao meio ambiente pelas atividades produtivas (Commoner, 1997). Assim como alguns autores consideram que a Produção Mais Limpa falha para atingir o desenvolvimento sustentável (Wallner, 1999), a Ecologia Industrial também é alvo de crítica. Por exemplo, Commoner (1997) considera que a produção industrial de compostos orgânicos sintéticos, que não são produzidos pela natureza como dioxinas e herbicidas, são inerentemente perigosos e medidas de segurança devem ser tomadas para proteger a ecosfera de seus efeitos. Este conceito tem uma conseqüência operacional: a produção em escala industrial destes compostos não deveria ser permitida. Este tipo de observação se deve, provavelmente, a que a relação processo produtivo/meio ambiente é uma questão que vem sendo abordada apenas recentemente e conceitos relativos ao tema estão, ainda, em fase de amadurecimento e aperfeiçoamento. Entretanto, a observação de Commoner é pertinente, pois a Ecologia Industrial não trata de forma clara deste tema.

Um dos grandes méritos da Ecologia Industrial é propor uma visão sistêmica que considera o sistema industrial como parte do sistema natural. O conceito de Eco-Tecnologia envolve a participação de duas ou mais empresas, com a finalidade de desenvolver/produzir produtos, resíduos e subprodutos cujo impacto no meio ambiente seja menor que aquele de cada empresa atuando independentemente de outras. O sucesso dependerá de um programa científico de pesquisa cujo alvo será o uso/reuso total de todos os constituintes do sistema inserido em um programa de gerenciamento ambiental implementado não só em cada indústria mas, principalmente, entre diferentes indústrias.

\section{Cenário Atual: exemplos de Produção Mais Limpa e Tecnologias Mais Limpas}

Exemplos focalizando a remoção de metais pesados de efluentes foram selecionados por uma série de razões: toxicidade extrema dos metais pesados, tendência a concentrar-se em tecidos animais e alto potencial carcinogênico. Neste artigo, se dará ênfase a sais de cromo utilizados em larga escala em todo o mundo, para transformação de peles de animais em couro. O couro curtido com cromo tem sido utilizado por quase um século para a manufatura de produtos como vestuário, luvas, calçados, estofados de automóveis, móveis e uma grande variedade de artefatos. O curtimento é a etapa mais importante na produção do couro e seu objetivo é interromper o processo de putrefação da pele (Heidemann, 1993). Durante o curtimento são geradas grandes quantidades de resíduos líquidos e sólidos que contém cromo e que são descartados no meio ambiente, especialmente na água e no solo.

Na Produção Mais Limpa reciclar, reutilizar, substituir ou desenvolver tecnologias (Tecnologias Mais Limpas) são alternativas que resultam na diminuição da quantidade de resíduo ou na produção de um subproduto com valor econômico. No caso da indústria de curtume, avaliações do processo com 
base na Produção Mais Limpa identificaram soluções que podem trazer benefícios econômicos e ambientais, por exemplo:

Aumento de temperatura e controle do $\mathrm{pH}$ do banho de curtimento aumenta a fixação do cromo nas peles ( Davies, 1979 e 1980);

Reciclar o cromo utilizado com reposição parcial do sal no banho de curtimento reduz a descarga de cromo no efluente (Gregori, 1991).

Geralmente, as Tecnologias Mais Limpas, que reduzem a quantidade de reagentes tóxicos descartados no ambiente, são simples e de fácil execução. Desta forma, a quantidade de efluente a ser tratado é menor. A implementação destas soluções resulta em aumento de produtividade e na melhora da qualidade dos produtos. Entretanto, não é possível recuperar todo o cromo dos banhos já utilizados, das águas de lavagem e do líquido retirado das peles antes da secagem. Por este motivo, são necessários tratamentos de efluentes líquidos para que o cromo não seja despejado no meio ambiente (Figura 1B).

\section{Estudos de Laboratório: possibilidades rumo às Eco-Tecnologias}

A literatura científica apresenta um grande número de tecnologias desenvolvidas para tratar efluentes contendo metais pesados. Pode-se classificar estas tecnologias para remediar e atenuar em duas diferentes categorias: estudos que se ocupam da remediação do solo e estudos dirigidos à remoção do metal de efluentes líquidos.

A contaminação do solo é um grande problema nos países industrializados, onde o metal descartado e dissolvido na fase líquida é absorvido pelo solo. Os métodos tradicionais geram grandes quantidades de lodo que contem metais pesados. Em outras palavras, os metais são removidos de solos contaminados e concentrados no lodo que deve ser guardado ou tratado e neutralizado. A Tabela 1 mostra alguns tratamentos propostos para remoção de metais pesados do solo.

A remoção dos metais na fonte, ou seja, dentro da empresa é uma alternativa que evita ou minimiza a utilização dos tratamentos convencionais. Por outro lado, os estudos da remediação do solo (Tabela 1) evidenciam que a grande capacidade de absorção do solo pode ser utilizada para retirar parte dos metais pesados presentes em sistemas aquáticos. Na Tabela 2 são apresentados trabalhos que exploraram esta abordagem.

Há, também, um grande número de estudos visando o desenvolvimento e utilização de novos materiais para tratar diretamente os efluentes industriais. Uma excelente revisão dos absorventes de baixo custo foi publicada por Bayley et al, 1999. Entre as substâncias investigadas se encontram materiais naturais como plantas (Lytle, 1998; Low, 1996), minerais (Moreira, 2001; Brigatti, 2000) (Tabelas 3 e 5), e muitos materiais desenvolvidos especialmente para remover metais de águas residuais como zeolitas (Mercier, 1997 e 1998) e carvão ativado (Bello, 1999; Ranganathan, 2000) (Tabela 4).

Outra abordagem, que vem surgindo, visa a utilização de resíduos de outros processos para tratar efluentes, por exemplo, o uso de raspas de madeira (Alves, 1993) e ganga mineral (Moreira, 2001; Buerge, 1999; Puls, 1999; Blowes, 1997; Zoubolis, 1995) (Tabela 5). Neste caso, a proposta dos pesquisadores resulta na minimização/reutilização de dois resíduos, transformando um dos resíduos em insumo para tratamento de efluentes. 
Os estudos citados (Tabelas 1 a 5) mostram que as conseqüências danosas aos seres vivos, impostas pela presença de metais pesados no ambiente, podem ser evitadas. Motivadas por este objetivo e com a finalidade de economizar água e insumos, as empresas devem desenvolver métodos de fabricação que levem em conta a diminuição da emissão de substâncias tóxicas no ambiente (Tecnologias Mais Limpas). Por outro lado, tratamentos inicialmente considerados como end-of-pipe podem ser utilizados de forma a promover a interação entre unidades industriais (Eco-Tecnologias).

Tab. 1. Diferentes métodos para descontaminação de solos

\begin{tabular}{|c|c|c|c|c|}
\hline metal & método & $\begin{array}{l}\text { absorvent } \\
\text { e }\end{array}$ & $\begin{array}{l}\text { Meio } \\
\text { tratado }\end{array}$ & Ref \\
\hline vários & eletroremediação & & solo & Acar et al, 1995 \\
\hline $\mathrm{Cu}$ & $\begin{array}{l}\text { eletroremediação }+ \text { membrana } \\
\text { seletiva de cátions }\end{array}$ & & areia & Li et al, 1998 \\
\hline $\begin{array}{l}\mathrm{Cu}, \mathrm{Cr}, \\
\mathrm{Hg}, \mathrm{Pb} \\
\text { e } \mathrm{Zn}\end{array}$ & eletroremediação & & solo arenoso & Hansen et al, 1997 \\
\hline $\mathrm{Cu}, \mathrm{Zn}$ & eletroremediação & & lodo industrial & Zagury et al, 1999 \\
\hline $\mathrm{Cr}$ & extração & $\mathrm{Na}_{4} \mathrm{P}_{2} \mathrm{O}_{7}$ & solo & $\begin{array}{l}\text { Pagilla e Canter, } \\
1999\end{array}$ \\
\hline $\mathrm{Cr}$ & vários & vários & solo & Bailey et al, 1999 \\
\hline $\mathrm{Cr}$ & $\begin{array}{l}\text { extração, oxidação, separação } \\
\text { e redução }\end{array}$ & $\begin{array}{l}\mathrm{H}_{2} \mathrm{SO}_{4} \\
\mathrm{H}_{2} \mathrm{O}_{2}\end{array}$ & $\begin{array}{l}\text { lama } \\
\text { curtume }\end{array}$ & Macchi et al, 1991 \\
\hline
\end{tabular}

Tab. 2. Diferentes métodos para descontaminação efluentes industriais com a utilização de solos

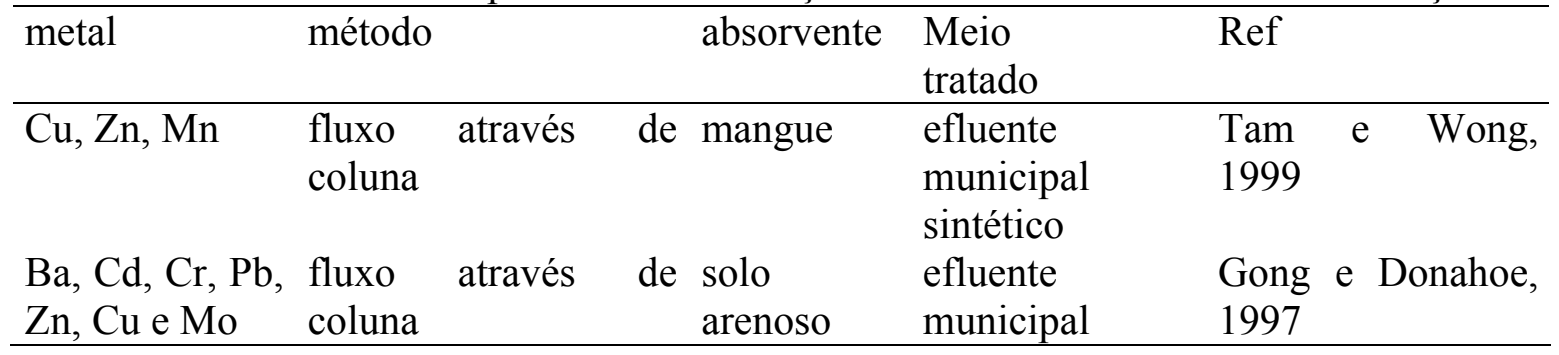

Tab. 3. Métodos para descontaminação de efluentes industriais com a utilização de materiais naturais

\begin{tabular}{|c|c|c|c|c|}
\hline metal & método & absorvente & $\begin{array}{l}\text { Meio } \\
\text { tratado }\end{array}$ & Ref \\
\hline $\mathrm{Cr}$ & fitoremediação/ absorção & $\begin{array}{l}\text { Jacinto } \\
\text { aquático }\end{array}$ & $\begin{array}{l}\text { efluente } \\
\text { curtume }\end{array}$ & de Lytle et al, 1998, \\
\hline $\mathrm{Cr}$ & fitoremediação/ absorção & musgo & $\begin{array}{l}\text { efluente } \\
\text { curtume }\end{array}$ & de Low et al, 1996 \\
\hline $\begin{array}{l}\mathrm{Co}, \quad \mathrm{Cu}, \\
\mathrm{Zn}, \mathrm{Cd}, \mathrm{Pb}\end{array}$ & leito fluidizado & sepiolita & $\begin{array}{l}\text { solução } \\
\text { sintética }\end{array}$ & Brigatti et al, 2000 \\
\hline
\end{tabular}


Tab. 4. Métodos para descontaminação de efluentes industriais com a utilização de materiais desenvolvidos para reter metais

\begin{tabular}{|c|c|c|c|c|}
\hline metal & método & absorvente & $\begin{array}{l}\text { Meio } \\
\text { tratado }\end{array}$ & Ref \\
\hline $\mathrm{Hg}^{2+}$ & absorção & $\begin{array}{l}\text { fluorohectorita } \\
\text { heterocíclica } \\
\text { mesoporosa }\end{array}$ & $\begin{array}{l}\text { solução } \\
\text { contendo } \mathrm{Hg}^{2+}\end{array}$ & $\begin{array}{l}\text { Mercier } \\
\text { Pinnavaia, } \\
1998\end{array}$ \\
\hline $\mathrm{Cr}, \mathrm{Hg}$ & batelada & carvão ativo & meio aquoso & Bello et al, 1999 \\
\hline
\end{tabular}

Tab. 5. Métodos para descontaminação de efluentes industriais com a utilização de resíduos de outros processos

\begin{tabular}{|c|c|c|c|c|}
\hline metal & método & absorvente & $\begin{array}{l}\text { Meio } \\
\text { tratado }\end{array}$ & Ref \\
\hline $\mathrm{Hg}^{2+}$ & adsorção & pirita & solução de $\mathrm{Hg}^{2+}$ & $\begin{array}{l}\text { Moreira et al, } \\
2001\end{array}$ \\
\hline $\mathrm{Cr}$ & $\begin{array}{l}\text { mistura com } \\
\text { borbulhamento de } \\
\text { ar }\end{array}$ & $\begin{array}{l}\text { casca de pinus } \\
\text { silvestre }\end{array}$ & $\begin{array}{l}\text { efluente de } \\
\text { curtume } \\
\text { (sintético) }\end{array}$ & $\begin{array}{l}\text { Alves et al, } \\
1993\end{array}$ \\
\hline $\begin{array}{l}\mathrm{Ag}, \quad \mathrm{Cd}, \\
\mathrm{Cr}, \quad \mathrm{Cu}, \\
\mathrm{Hg}, \quad \mathrm{Ni}, \\
\mathrm{Pb}, \quad \mathrm{U} \quad \mathrm{e} \\
\mathrm{Zn}\end{array}$ & absorção & $\begin{array}{l}\text { casca de carvalho, } \\
\text { faia, } \\
\text { vermelhos e pinus } \\
\text { silvestre }\end{array}$ & $\begin{array}{l}\text { Solução } \\
\text { sintética }\end{array}$ & $\begin{array}{l}\text { Gaballah } \\
\text { Kilbertus, } \\
1997\end{array}$ \\
\hline $\mathrm{Cr}$ & redução & $\mathrm{Fe}(\mathrm{II})$ & $\begin{array}{l}\text { água (área } \\
\text { industrial) }\end{array}$ & $\begin{array}{l}\text { Buerge e Hug, } \\
1999\end{array}$ \\
\hline $\mathrm{Cu}, \mathrm{Cd}$ & adsorção, redução & ferro & água & Shokes e \\
\hline $\mathrm{Zn}, \mathrm{Ni}$ & precipitação & & mineração & Moller, 1999 \\
\hline $\mathrm{Cr}$ & barreira permeável & ferro e areia & $\begin{array}{l}\text { efluente } \\
\text { cromeação }\end{array}$ & $\begin{array}{l}\text { Puls et al, } \\
1999\end{array}$ \\
\hline $\mathrm{Cr}$ & barreira permeável & $\begin{array}{l}\text { ferro metálico, } \\
\text { siderita e pirita com } \\
\text { areia de quartzo }\end{array}$ & $\begin{array}{l}\text { solução } \\
\text { sintética }\end{array}$ & $\begin{array}{l}\text { Blowes et al, } \\
1997\end{array}$ \\
\hline $\begin{array}{l}\mathrm{Cu}, \quad \mathrm{Zn}, \\
\mathrm{Ni}, \mathrm{Cd}\end{array}$ & batelada e coluna & lama vermelha & efluente urbano & $\begin{array}{l}\text { Lopez et al, } \\
1998\end{array}$ \\
\hline $\mathrm{Cr}$ & adsorção & pirita & $\begin{array}{l}\text { solução } \\
\text { sintética }\end{array}$ & $\begin{array}{l}\text { Zoubolis et al, } \\
1995\end{array}$ \\
\hline
\end{tabular}

\section{Proposta de APLICAÇão}

Nos curtumes, os esforços para minimizar o descarte de cromo no ambiente estão fortemente ligados à otimização do uso do cromo (como mencionado na seção Cenário atual: exemplos de Produção Mais Limpa e Tecnologias Mais Limpas): melhora da fixação do cromo, reciclagem dos banhos de curtimento e reutilização das águas de lavagem. Por outro lado, é inevitável que plantas de tratamento de efluentes sejam instaladas para limpar a água que será despejada nos sistemas aquáticos. Em alguns casos, estas plantas de tratamento atendem a mais de um curtume, como é o caso do distrito industrial de Franca, no interior de São Paulo. O emprego de Eco-Tecnologias pode, neste caso, ser 
adequado. A Tabela 5 mostra trabalhos que permitem propor a utilização de resíduos para minimizar a emissão de metal pesado pelos curtumes: a combinação de dois resíduos faria com que dois problemas ambientais fossem atenuados. Ressalta-se que, dos estudos que utilizam resíduos de outros processos, o trabalho de Moreira et al. (2001) emprega amostra mineral brasileira comum em ganga de mineração, inclusive na indústria de extração do carvão.

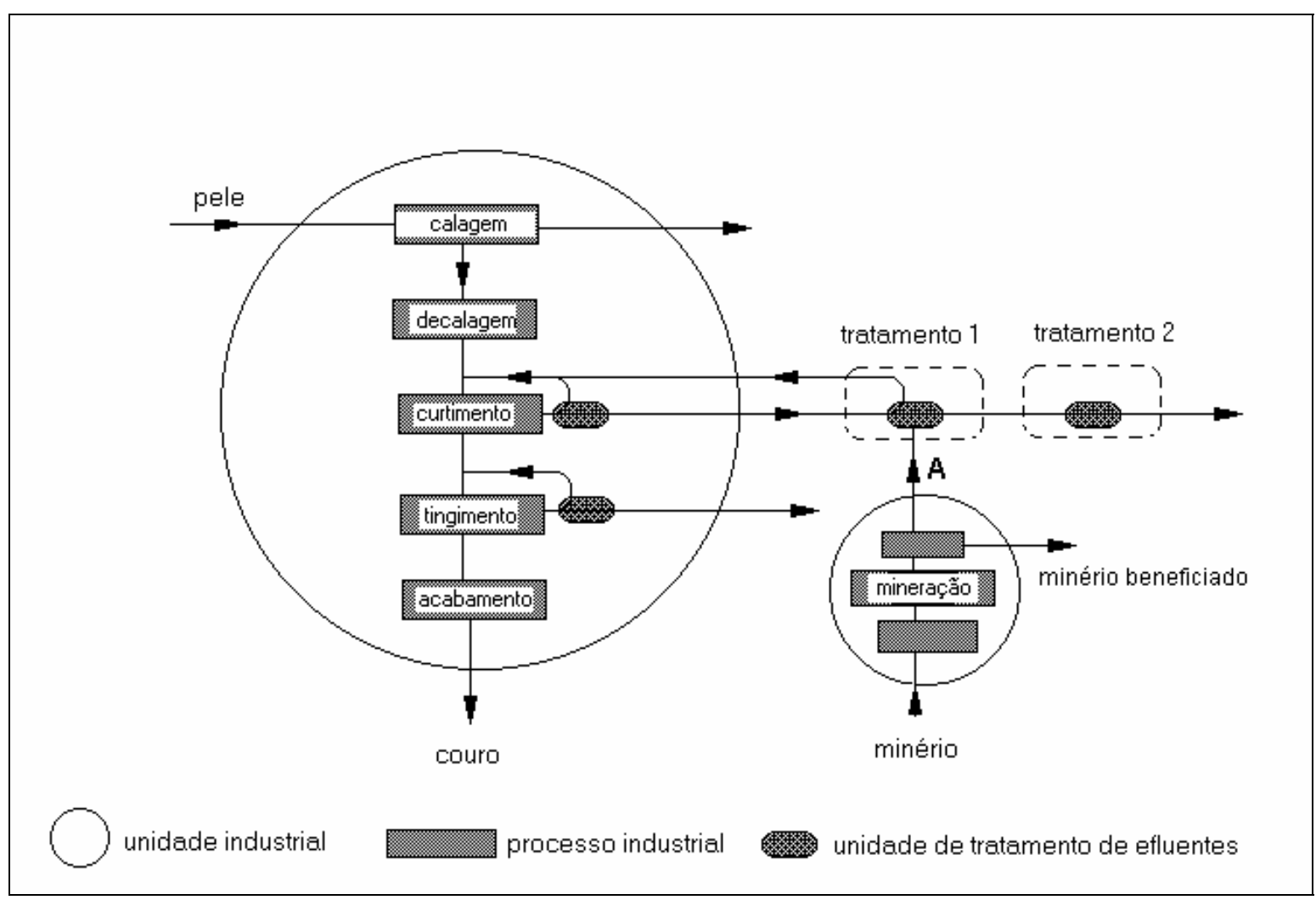

Fig. 3. Representação da combinação de dois setores industriais, mineração e curtume, ligados por uma Eco-Tecnologia. No fluxo A representa-se o resíduo do beneficiamento do carvão usado no tratamento de efluentes (tratamento 1 - retirada de cromo que retorna para o processo de curtimento).

O impacto ambiental da indústria de mineração é, em grande parte, devido à drenagem ácida, já que os resíduos descartados continuam a causar problemas ambientais por centenas de anos, após o término da vida econômica da mina (Souza, 2001). Durante o beneficiamento do carvão de 30 a $60 \%$ do material minerado é refugado, resultando na produção de grandes volumes de rejeitos, constituídos basicamente por materiais carbonosos e minerais (pirita e argilo-minerais) sem valor comercial, que são depositados em áreas próximas ao local de mineração (Souza, 2001). A exposição dos resíduos de mineração (pirita) ao ambiente resulta em alto impacto ambiental negativo devida à dissipação dos produtos da ação da intempérie, que contém ácidos. O desafio, neste caso, é estabelecer um controle efetivo e economicamente viável para deter a oxidação desta ganga ou encontrar uma forma de utilizar estes resíduos para que não sejam deixados a céu aberto.

A utilização de resíduos de mineração para tratar efluentes de curtume (Figura 3 - tratamento 1) pode atenuar a drenagem ácida devida ao descarte de sulfetos minerais enquanto auxilia na remoção do cromo de efluentes de curtume (Buerge, 1999; Puls, 1999; Blowes, 1997; Zoubolis, 1995). Em paralelo, deve-se desenvolver formas para recuperar o cromo retirado que permitam a reutilização dos sais de cromo no próprio curtume. Pode-se ainda complementar o tratamento com outras Eco- 
tecnologias como fitoremediação (Lytle, 1998; Low, 1996) (Tabela 3) ou o desenvolvimento de materiais para garantir a qualidade final da água a ser despejada nos sistemas fluviais (Figura 3 tratamento 2) (Tabela 4).

Esta proposta requer uma ação integrada do setor industrial em relação ao meio ambiente, ao contrário do que é feito atualmente, quando cada empresa procura reduzir os efeitos danosos de processos de forma isolada. Cabe lembrar que, a transferência de resíduos, além do custo do transporte, poderia causar um problema ambiental adicional. Portanto, este tipo de abordagem implica na procura de soluções regionais. Desta forma, considerando o exemplo mineração/curtume, a proximidade das duas atividades deve ser decisiva.

Condição favorável é encontrada na região sul do Brasil. As reservas de carvão brasileiras representam uma porção significativa das fontes de energia não renovável do país e os maiores depósitos estão localizados no Rio grande do Sul e em Santa Catarina (Figura 4). O Paraná tem, também, alguns depósitos carboníferos menores. Até agora, apenas uma parcela destas reservas tem sido explorada, com o crescimento do consumo energético, a tendência é incrementar o uso do carvão como fonte de energia mais barata. $\mathrm{O}$ total estimado de reservas é de 32 bilhões de toneladas sendo que 87 \% encontra-se no Rio Grande do Sul (Centro de Ecologia/UFRGS, 2000).

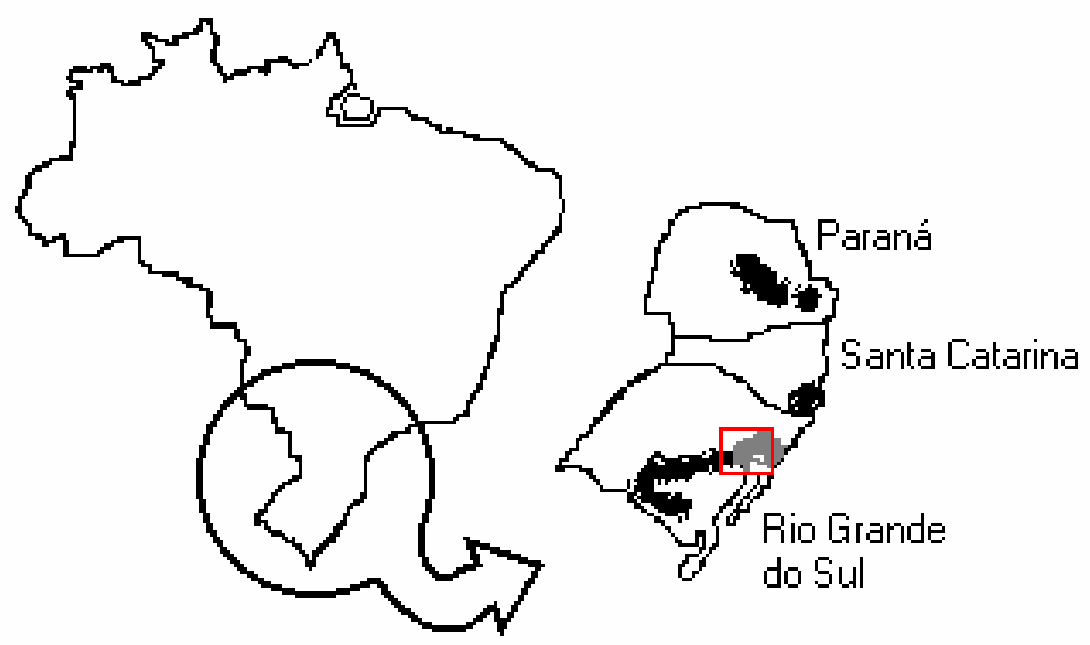

reservas de carvẫo

regiẫo de curtumes

Fig. 4. Localização das reservas de carvão no sul do Brasil e da região de curtumes. A diagonal do quadrado tem aproximadamente $200 \mathrm{~km}$, e encerra a região de maior produção de carvão e maior concentração de curtumes.

Por outro lado, uma parte significativa da indústria nacional de calçados se localiza na mesma região. Várias manufaturas e curtumes têm sua sede no Vale dos Sinos: na Figura 4 o quadrado vermelho inclui a região de maior produção de carvão e de maior concentração de curtumes. Desta forma, seria razoável propor que a cooperação mineração/curtume, com base nos estudos de laboratório citados neste trabalho, poderia resultar na implementação de uma Eco-Tecnologia na fronteira entre 
estes dois setores industriais. O exemplo mineração/curtume aqui citado é uma de muitas alternativas. Na mesma região, a utilização de resíduos da agricultura para tratar os efluentes dos curtumes também seria viável. Para isto, serão necessários estudos com resíduos agrícolas locais. Estudos feitos na Europa por Gaballah e Kilbertus (1997) e Alves et al. (1993) (Tabela 5) são exemplo deste tipo de alternativa.

È importante destacar que benefícios surgem da implantação de uma Eco-Tecnologia. De uma forma esquemática, podem ser identificados benefícios para o setor industrial, para o meio ambiente e para a sociedade.

Para as companhias envolvidas (mineração/curtume), a Eco-Tecnologia oferece a oportunidade de diminuir custos: o resíduo de mineração é retirado do ambiente e transformado em insumo para tratamento de efluente, a indústria de curtume dispõe de insumo de menor custo que os convencionais para tratamento de seus efluentes. Além disto, esta prática evitaria penalizações por parte de órgãos de controle ambiental e os gastos com perdas financeiras futuras para remediação de áreas degradadas. Vale lembrar que o custo para reabilitar áreas degradadas é estimado como sendo de 10 a 50 vezes maior que o custo das medidas de prevenção (Banco Mundial, 1992). A interação entre empresas leva ao compartilhamento de despesas. Por exemplo, (i) a mineração, se isolada, arcaria com o custo de remoção e de combate à drenagem ácida; (ii) a indústria de curtume arcaria totalmente com o custo de aquisição e transporte de insumos para retirada do cromo; (iii) no caso da utilização de Eco-Tecnologia, ambas dividiriam parte dos custos envolvidos em (i) e (ii).

Como benefício ambiental temos a redução de resíduos e de poluentes. Pode-se considerar que o benefício mais importante é a diminuição de demanda de recursos naturais, pela reutilização de resíduos. Estes benefícios estariam contribuindo para se atingir o desenvolvimento sustentável (ver Nota 5, item ii).

A provável melhora da performance econômica das empresas envolvidas trará desenvolvimento econômico à comunidade local. Este tipo de interação entre empresas possibilitará novos empreendimentos regionais, criando novos postos de trabalho, gerados, direta ou indiretamente, pela Eco-Tecnologia. A redução dos resíduos sólidos e metais tóxicos nos efluentes líquidos reduz gastos públicos com infra-estrutura e manutenção de plantas de tratamento de águas e esgotos. A qualidade de vida local, melhorada ou conservada, diminuirá gastos na área de saúde pública.

Cabe ressaltar que a interação entre diferentes setores industriais terá dificuldades e encontrará resistência. O sucesso na implementação de Eco-Tecnologias dependerá de um novo tipo de colaboração, entre instituições públicas e empresas locais, e de uma nova visão dos profissionais envolvidos. Inicialmente, o processo de implementação trará custos adicionais, por exemplo com treinamento de pessoal, o que pode causar relutância. Pequenas e médias empresas (como a maioria dos curtumes) terão dificuldade para acessar estas novas formas de gerenciamento e de conhecimento tecnológico. Cabe aos orgãos públicos, aos sindicatos patronais e às universidades dar suporte a estas empresas. Provavelmente, a maior dificuldade será mudar a percepção de algumas empresas (grandes e pequenas) quanto ao conceito de interdependência. Outra dificuldade está em que, no Brasil, ainda não existem estruturas passíveis de levar a uma verdadeira repartição de trabalho nem no cumprimento das tarefas públicas. Há, também, falta de cooperação entre municípios (horizontal) e entre estados e municípios (vertical) (Centro de Ecologia/UFRGS, 2000). Sob a ótica da Ecologia Industrial é importante visualizar que a cooperação é uma forma de aumentar a vantagem competitiva regional. 


\section{CONCLUSÃo}

Definições de conceitos fundamentais e exemplos de Produção Mais Limpa foram apresentados com a finalidade de contrapor abordagens e ilustrar a atual forma utilizada para minimização do impacto ambiental na indústria de curtume. Foi realizada uma revisão da literatura técnico-científica mostrando tecnologias não convencionais para mostrar o estado da arte na remoção de metais de efluentes líquidos. Após a discussão dos conceitos e da análise da literatura, foi possível propor o uso de resíduos da indústria de mineração para o tratamento de efluentes da indústria de curtume na região sul do Brasil.

Cabe agora, com base na discussão apresentada, responder algumas questões de ordem geral:

(i) qual a relação entre as Tecnologias Mais Limpas e o novo conceito de Eco-Tecnologias?

As Tecnologias Mais Limpas são uma das atividades ou ferramentas da Produção Mais Limpa, que se aplicam aos processos de fabricação e manufatura considerando a melhor integração entre os subsistemas de produção para maximizar a eficiência na produção com relação à utilização de insumos e à produção de resíduos e minimizar os danos ambientais. Por outro lado, a Eco-Tecnologia, como atividade da Ecologia Industrial, envolve a participação de duas ou mais empresas. A implantação desta atividade é feita no sentido de diminuir o impacto no meio ambiente, com a característica de causar um impacto menor que aquele de cada empresa atuando independentemente de outras. A interação entre empresas, além dos benefícios ambientais resultantes da aplicação de Eco-Tecnologias, deve resultar em ganhos econômicos e sociais.

Destes conceitos, percebe-se que a transição de uma Tecnologia Mais Limpa para uma EcoTecnologia implica necessariamente na integração entre diferentes empresas. Este trabalho, fundamentado em uma revisão da literatura, mostra que uma visão sistêmica das características regionais é imprescindível para que a transição ocorra. Neste sentido, foi apresentado o potencial de aplicação de Eco-Tecnologia na região sul do país. Certamente, estudos semelhantes podem identificar outras regiões com potencial para implementação da Ecologia Industrial no Brasil. Observou-se que os estudos referentes à tecnologias não convencionais de remediação podem oferecer novas opções para prevenção e controle da poluição mas, principalmente, podem ser empregados para interligar processos e empresas. A visão sistêmica sob a ótica da Ecologia Industrial indica, claramente, a necessidade de esforços do setor acadêmico no sentido de contribuir para o desenvolvimento e a implementação de Eco-Tecnologias.

(ii) como as tecnologias desenvolvidas em ambiente de laboratório podem se tornar EcoTecnologias?

As soluções desenvolvidas em ambiente de laboratório ainda estão dirigidas para problemas específicos de poluição. Entretanto, a combinação destes estudos com conceitos de Ecologia Industrial pode levar à implementação de Eco-Tecnologias entre unidades industriais. Desta forma, pode-se considerar estes estudos acadêmicos como o primeiro passo em direção às Eco-Tecnologias.

Para finalizar, é bom ressaltar que há grande responsabilidade na implementação e no desenvolvimento destes conceitos, que hoje estão, especialmente, nas mãos de pesquisadores, educadores, administradores e empresários. Os pesquisadores deverão gerar e divulgar o conhecimento técnico e científico, desenvolvendo os recursos necessários. Os educadores terão a responsabilidade de incentivar atitudes e criar uma visão adequada do futuro. Administradores, do setor público e privado, e 
empresários terão a tarefa de aplicar estes conceitos gerenciando os recursos disponíveis. Gerenciar o meio ambiente local significa conhecer as características, potencialidades e limitações da região para melhor orientar o desenvolvimento por meio da atração de atividades econômicas - ou incentivos a aquelas já implantadas - compatíveis com a vocação ecológica e com a capacidade real para sua sustentabilidade.

\section{Agradecimentos}

Esta pesquisa foi desenvolvida no projeto que estuda a cadeia produtiva carne-couro-calçados como parte do Programa de Mestrado em Engenharia de Produção da UNIP. Os autores agradecem os comentários e sugestões dos assessores científicos desta revista, que enriqueceram o texto. Resultados experimentais referentes ao uso de ganga mineral para remoção de metais pesados de efluentes fazem parte do Projeto FAPESP 95/9333-2. O apoio da FAPESP é especialmente reconhecido.

\section{Artigo recebido em 31/08/2001. Aprovado em 14/11/2002.}

\section{REFERÊNCIAS BIBLIOGRÁFICAS}

ACAR, C.A. Electrokinetic remediation: basis and technology status. J. Hazardous Mat. 40 (1995), p. 117-137.

ALVES, M. M., GONZALES BEÇA, C. G., CARVALHO, R. G., CASTANHEIRA, J.M., PEREIRA, M. C. S. , VASCONCELOS, L.A.T. Chromium removal in tannery wastewaters polishing by pinus silvestris bark. Wat. Res. 27 (1993), p.1333-1338.

AYRES, R. U., NORBERG-BOHM, V., PRINCE, J., STIGLIANI, W. M., YANOWITZ, J., Industrial Metabolism, the environment and application of materials balance principles for selected chemicals. Research Report RR-89-11, IIASA, Laxemburg, 1989.

BAILEY, S. E., OLIN, T. J., BRICKA, R. M., ADRIAN, D. D. A review of potentialy low-cost sorbents for heavy metal. Wat. Res. 38 (1999), p.2469-2479.

Banco Mundial. World Development Report: Development and Environment. New York, Oxford University Press, 1992.

BELLO, G., CID, R., GARCIA, R., ARRIAGADA, R. Retention of Cr(VI) and Hg(II) in Eucalyptus globulus-and peach stone-activated carbons. J. Chem. Tech. Biotechnol. 74 (1999), p. 904-910.

BLOWES, D. W., PTACEK, C. J., JAMBOR, J. L. In-situ remediation of Cr(VI)-contamined groundwater using permeable reactive walls:labotarory studies. Environ. Sci. Tech. 31 (1997), p. 33483357.

BRIGATTI, M. F., LUGLI, C., POPPI, L. Kinetics of heavy metal removal and recovery in sepiolite. Appl. Clay Sci.16 (2000), p. 45-57.

BUERGE, I. J., HUG, S. J. Influence of mineral surfaces on Chromium (VI) reduction by iron (II). Environ.Sci.Tech. 33 (1999), p. 4285-4291. 
Centro de Ecologia/UFRGS. Carvão e Meio Ambiente. Porto Alegre, Ed. Universidade, 2000.

COMMONER, B. The relation between industrial and Ecologycal Systems. J. Cleaner Prod., 5 (1997), p. 125-129.

DAVIS, M. H., Scroggie J. G.. Theory and Practice of Direct Chrome Liquor Recycling, Das Leder, 31 (1980), p. 1-8.

DAVIS, M. H., SCROGGIE J. G., Theory and practice of direct chrome liquor recycling, XVI International Union of Leather Technologists and Chemists Societies Congress, Versailles-France, v. II. (1979), p.4-6.

FROSCH, R.A., GALLOPOULOS N.E. Strategies for Manufacturing. Scientific American, 1989.

GABALLAH, I., KILBERTUS, G. Recovery of heavy metals ions through decontamination of synthetic solutions and industrial effluents using modified barkas. J. Geochem. Expl. 62 (1997), p. 241286.

GONG, C, DONAHUE, R. J. An experimental study of heavy metal attenuation and mobility in sandy loam soils. Appl. Geochem. 12 (1997), p. 243-254.

GREGORI, J., MARSAL, A., MANICH, A. M., Cot, J., Optimización del proceso de curtición al cromo: influencia del comportamiento de tres tipos de agentes complejantes, XXI International Union of Leather Technologists and Chemists Societies Congress, Barcelona-Spain, 25-29 September 1991.

HANSEN, H. K., OTTOSEN, L. M., KLIEM, B. K., VILLUMEN, A. Electrodialytic remediation of soils polluted with $\mathrm{Cu}, \mathrm{Cr}, \mathrm{Hg}, \mathrm{Pb}$ and Zn.J. Chem. Technol. Biotechnol. 70 (1997), p. 673.

HEIDEMANN, E., Vergleich zwischen Chrom und Vegetabilgerbung, abgeleitet aus den Bindepositionen am Kollagen, Das Leder, 5(1993), p. 99-104.

LI, Z. YU, Ji-Wei, NERETNIEKS, I. Electroremediation: Removal of heavy metals from soils by using cation selective membrane. Environ. Sci. Technol. 32 (1998), p. 394-397.

LOPEZ, E., SOTO, B., ARIAS, M., NUÑEZ, A., RUBINOS, D., BARRAL, T., 1998. Adsorbent properties of red mud and its use for wastewater treatment. Wat. Res. 32 (), p. 1314-1322.

LOW, K.S., LEE, C.K., TAN, S.G. Sorption of trivalent chromium from tannery waste by moss. Environ. Tech. 18 (1996), p. 449-454.

LYTLE, C. L., LYTle, F. W., YANG, N., QIAN, J. H., HANSEN, D., ZAYED, A. TERRY, N. Reduction of $\mathrm{Cr}(\mathrm{VI})$ to $\mathrm{Cr}(\mathrm{III})$ by wetland plants: potential for in situ heavy metal detoxification. Environ. Sci. Technol. 32 (1998), p. 3087-3093.

MACCHI, G., PAGANO, M., PETTINE, M., SANTORI, M., TIRAVANTI, G. A bench study on chromium recovery from tannery sludge. Wat. Res. 25 (1991), p. 1019-1026. 
MERCIER, L., PINNAVAIA, T. J. Access in mesoporous materials: Advantages of a uniform pore structure in the design of a heavy metal ion adsorbent for environmental remediation. Adv. Mat. 9 (1997), p. 500-503.

MERCIER, L., PINNAVAIA, T. J. A functionalized porous clay heterostructure for heavy metal ion $\left(\mathrm{Hg}^{2+}\right)$ traping. Microporous and Mesoporous Materials 20 (1998), p. 101-106.

MOREIRA, W.A., GIANNETTI, B. F., BONILlA, S. H., ALMEIDA, C. M. V. B., RABÓCZKAY, T. Use of pyrite as an attenuation strategy for ions mercury traping. Proceedings of the VI Southern Meeting on Mineral Technology, 2 (2001) p. 521-526.

PAGILLA, K. R., CANTER, L. W. Laboratory studies on remediation of chromium-contamined solis. J. Environ. Eng. 125 (1995), p. 243-248.

PULS, R.W., PAUL, C.J., POWELL, R.M. The Application of in siti permeable reactive (zero-valent iron) barrier technology for the remediation of chromate-contaminated groundwater. Appl. Geochem. 1454 (1999), p. 989-1000.

RANGANATHAN, K. Chromium removal by activated carbons prepared from Casurina equisetifolia leaves. Bios. Tech. 7354 (2000), p. 99-103.

SHOKES, T. E., MOLLER, G. Removal of dissolved heavy metal from acid rock drainage using iron metal. Environ. Sci. Tech. 3354 (1999), p. 282-287.

SOUZA, V.P., SOARES, P. S. M., RODRIGUES-FILHO, S. Proceedings of the VI Southern Meeting on Mineral Technology, 2 (2001) p. 603-608.

TAM, N. F. Y., WONG, Y. S. Mangrove soils in removing pollutants from municipal wastewater of different salinities. J. Environ. Qual. 2854 (1999), p. 556-562.

WALLNER, H.P. Towards sustainable development of industry: networking, complexity and ecoclusters. J. Cleaner Prod., 7 (1999), p. 49-58.

ZOUBOLIS, A. I., KYDROS, K. A., MATIS, K. A. Removal of hexavalent chromium anions from solutions by pyrite fines. War. Res. 2954 (1995), p.1755-1760.

\section{NOTAS}

\section{${ }^{1}$ Ecologia Industrial}

Há muitas definições de Ecologia Industrial, aqui são apresentadas duas das mais significativas histórica e conceitualmente:

"De forma quase teológica, Ecologia Industrial é o meio pelo qual a humanidade pode, deliberada e racionalmente, atingir e manter o desenvolvimento sustentável, com contínua evolução econômica, cultural e tecnológica. O conceito consiste de uma visão sistêmica da atividade econômica e suas interrelações com os sistemas fundamentais biológicos, químicos e físicos, com a finalidade de estabelecer e manter a espécie humana em um nível que pode ser sustentado indefinidamente." - TE Graedel and BR Allenby, "Industrial Ecology”, Prentice Hall, 1995. 
"A idéia da Ecologia Industrial se baseia na analogia com os sistemas naturais. Na natureza, um sistema ecológico opera em uma rede de conexões, na qual os organismos vivos consomem outros organismos e/ou seus resíduos. A estrutura de um sistema natural e a estrutura de um sistema industrial, ou um sistema econômico, são extremamente semelhantes." - Robert A. Frosh, "Industrial Ecology: A Philosophical Introduction”, Proceedings of the National Academy of Sciences , 1992, USA.

${ }^{2}$ Tecnologias Mais Limpas

São técnicas genéricas para implementação da Produção Mais Limpa incluem práticas de manutenção, otimização de processos, substituição de matéria prima, novas tecnologias e novo projeto. Além de preservar valores ambientais, uma finalidade desta ferramenta da Produção Mais Limpa é de prevenir o uso ineficiente de matérias primas e reduzir custos operacionais, de tratamento e de descarte - UNEP (United Nations Environment Program).

${ }^{3}$ Produção Mais Limpa

De acordo com a UNEP (United Nations Environment Program), TIE (Division of Technology, Industry and Environment) que cunharam o termo em 1989, a Produção Mais Limpa é a aplicação contínua de uma estratégia integrada de prevenção ambiental a processos, produtos e serviços para aumentar a eficiência de produção e reduzir os riscos para o ser humano e o ambiente. A Produção Mais Limpa é uma filosofia proativa, que antecipa e prevê possíveis impactos e pode ser aplicada não só a processos de produção mas, também, ao longo de todo o ciclo de vida de um produto, desde a fase de projeto, passando pela fase de consumo até sua disposição final. Visa conservar fontes de matéria prima, eliminação de insumos tóxicos e a redução de resíduos e emissões.

${ }^{4}$ End-of-pipe

O meio tradicional de combate à poluição é o emprego de sistemas end-of-pipe, isto é, o tratamento de resíduos e efluentes. Os sistemas end-of-pipe podem incluir o tratamento de água, ar e resíduos sólidos. As mais diversas tecnologias foram desenvolvidas com este objetivo, como sistemas químicos e biológicos para tratamento de água e aterros para resíduos sólidos. Para cada efluente haverá, provavelmente, várias opções de tratamentos, igualmente aceitáveis, com diferenças na qualidade, no custo e na performance ambiental. -TE Graedel and BR Allenby, "Industrial Ecology", Prentice Hall, 1995.

${ }^{5}$ Desenvolvimento sustentável

Há diversas definições sobre o que é desenvolvimento sustentável, já que este é um conceito que abriga uma multiplicidade de entendimentos. Apresenta-se as definições que representam marcos históricos.

Desenvolvimento sustentável é um termo criado em 1987, no Relatório Nosso Futuro Comum da "Brundtland Commision" (Comissão Mundial para Meio Ambiente e Desenvolvimento) como: o "desenvolvimento que satisfaz as necessidades do presente sem comprometer a capacidade das futuras gerações de satisfazer as suas próprias necessidades". Ou seja, as gerações presentes têm o dever de deixar, para as gerações futuras, uma natureza intacta. A economia, até então, baseada nos parâmetros trabalho e capital, passa a considerar em terceiro parâmetro: o capital natural.

Para se atingir o desenvolvimento sustentável deve-se observar dois princípios básicos: (i) a velocidade de retirada de recursos naturais deve ser igual à velocidade de regeneração (rendimento sustentável) e 
CIÊNCIA E TECNOLOGIA - IMPLEMENTAÇÃO DE ECO-TECNOLOGIAS RUMO A ECOLOGIA INDUSTRIAL Biagio Fernando Gianetti - Cecília M. Villas Boas de Almeida - Silvia H. Bonilla

(ii) a velocidade de produção de resíduos deve ser igual à capacidade do ambiente para absorver estes resíduos. - Daly H. E. (Toward Some Operational Principles os Sustainable Development, Ecological Economics, 1990, v.2,p. 1-6) 